Goal Available data suggest that SARS-Cov-2 infection is usually mild in children. The aim of this study was to describe clinical and biological features and outcome of Covid 19 in Tunisian infants and newborns.

Method We reviewed files of patients $<12$ months, diagnosed with Covid 19 and hospitalized in pediatric ' $C$ ' department of Béchir Hamza children's hospital between 15 March 2020 and 15 April 2021.

Results We enrolled thirteen infants and newborns: 11 boys and 2 girls.

Median age was 3 months [10 days; 15 months]. Five were aged between 10 and 28 days, seven were aged between 1 and 12 months. Three patients had serious underlying diseases: two cases of interventricular communication and one case of myelomeningocele and meningitis. Six patients had exposure with persons confirmed to Covid 19. Most frequent signs were: fever (11/13), diarrhea and vomiting (3/13), cough (3/13), nasal congestion and grunting (2/13).

On admission, physical examination found high fever in seven cases. Two infants had marbled skin and one newborn had jaundice. Two patients had tachypnea. Median heart rate was 135. Three patients had Oxygen saturation $<94 \%$.

Laboratory testing found median white blood cell count 9496/mm3 [3980-17200], median neutrophil count 4540/mm3 [480-10260], median lymphocyte count 3370/mm3 [9908020], median monocyt count 1701/mm3 [990-3370] and median C-reactive-protein 4,45mg/l [0-16]. The patient who had jaundice had total bilirubin level at $182 \mu \mathrm{mol} / \mathrm{L}$ and unconjugated bilirubin level at $169 \mu \mathrm{mol} / \mathrm{L}$. Five patients had lumbar puncture and were all negative.

All patients received symptomatic treatment. Two patients received oxygen through nasal canula and one patient was put on non invasive ventilation. As we received the result of RT PCR, all patients were admitted in isolation ward.

Median duration of symptoms was 3 days [3-7 days]. Median hospital stay was 3 days [2-7 days]. Death occurred one patient with interventricular communication. He had cardiogenic shock 3 days after his diagnosis with Covid 19.

Conclusion Prevalence of Covid 19 is less in children than in adults. In our case serie of 13 infants, five were newborns. Contamination was caused mostly by another family member. Fever, cough and gastrointestinal signs were the most common symptoms. One death occurred in an infant with interventricular communication. None of our patient had multisystem inflammatory syndrome after his infection.

\section{MULTISYSTEM INFLAMMATORY SYNDROME DUE TO SARS-COV-2 (MIS-C) IN TUNISIAN CHILDREN: A SINGLE- CENTER EXPERIENCE}

Rania Ben Rabeh*, Nada Missaoui, Salem Yahyaoui, Sofiène Atiattalah, Msaddek Assidi, Sonia Mazigh, Samir Boukthir. Béchir Hamza Children's Hospital

\subsection{6/archdischild-2021-europaediatrics.351}

Goal Acute corona virus disease (COVID-19) appears mild in children in comparison to adults. But multisystem inflammatory syndrome in children caused concerns among pediatricians since its first description in May 2020.

In this paper we describe a Tunisian experience in management of MISC-C.

Method We reviewed medical files of all children diagnosed with MIS-C and admitted in the pediatric C department of
Bechir Hamza Children's Hospital of Tunis. We analysed demographic, medical and biological features.

Results We enrolled 11 patients. The first one was admitted in September 2020, weeks after the beginning of the second wave of Covid 19 in Tunisia.

Median age was 5 years, nine of them were boys. One patient had neonatal sclerosing cholangitis. The most common symptoms were: fever (11/11), gastrointestinal symptoms (8/ 11), rash, conjunctivitis and cheilitis (5/11).

Five patients had exposure with a confirmed family member. Physical examination at admission found high fever $(\mathrm{n}=10)$ and tachypnea $(\mathrm{n}=8)$ with a median respiratory rate at 30. Median heart rate was 110 and median oxygen saturation was 99\%. Four patients had abdominal tenderness. During hospital stay, one patient presented generalized seizure due to multisystem failure.

Laboratory testing found medium white blood cell count $10820 / \mathrm{mm} 3$, neutrophil cell count $8463 / \mathrm{mm} 3$, lymphocyte $1507 / \mathrm{mm} 3$ (6 patients had lymphopenia <1500), median Creactive-protein level was $177 \mathrm{mg} / \mathrm{l}[83-322 \mathrm{mg} / \mathrm{l}]$ and median erythrocyte sedimentation rate was $65 \mathrm{~mm}$ [42-130 mm]. Five patients had elevated troponin level. Median D-dimer level was $1,8 \mathrm{mg} / \mathrm{l}$ and median fibrinogen level was $4,5 \mathrm{~g} / \mathrm{l}$. One patient had positive SARS-Cov-2 RT-PCR and the others had positive serology. Four patients presented heart dysfunction and received vasopressive support. Coronary dilatation was found in four patients. Three patients were admitted in ICU, all of them had cardiogenic shock. One patient was put under mechanical ventilation. One patient presented fulminant liver failure and macrophage activation syndrome. One patient presented coronavirus-associated nephropathy with nephrotic syndrome. All patients received immunoglobulin infusion, eight received systemic glucocorticoids. Median length of hospital stay was 12 days $[5 ; 33$ days]. No death occured.

Conclusion MIS-C is a severe result of SARS-Cov-2 infection. Cardiovascular dysfunction is the most frequent complication. Treatment is based on immunoglobulin infusion, systemic glucocorticoids and management of cardiac dysfunction. Management of these children has high cost, especially in developing countries like Tunisia.

\section{MAKING EDUCATIONAL VR VIDEO GAMES ACCESSIBLE TO ALL AGE DEMOGRAPHICS IN THE MEDICAL FIELD}

${ }^{1}$ Zachary Tan, ${ }^{2}$ Conor Tapley, ${ }^{2}$ William Hulshof*, ${ }^{1}$ Alfonso Rodriguez Herrera. ' St. Luke's General Hospital; ${ }^{2}$ Technological University Dublin

10.1136/archdischild-2021-europaediatrics.352

Our objectives were:

- To test how different age demographics of medical professionals interacted with our VR video game.

- Record what issues (if any) they faced or had while doing so.

- Analyse what possible solutions could be implemented to make VR video games more accessible to all age demographics in the medical field.

Due to the circumstances created by the COVID-19 pandemic, some of our data came from previous surveys and playtests we created for our game. As well as this, we put our game and a new survey for it up on Itch.io. Itch.io is a public website where people can download free/purchasable games. Our survey questioned individuals on various topics 